\title{
Kinetics and Mechanism of Oxidation of Aniline by $N$-Bromophthalimide
}

\author{
N. M. I. ALHAJI", G. K. AYYADURAI ${ }^{\mathrm{a}}$ and A. SHAJAHAN ${ }^{\mathrm{b}}$ \\ *P.G. \& Research Department of Chemistry, Khadir Mohideen College, Adirampattinam, \\ Tamilnadu-614701, India \\ ${ }^{a}$ Department of Chemistry, Sri Sai Ram Engineering College, West Tambaram, Chennai, \\ Tamilnadu-600044, India \\ ${ }^{\mathrm{b}}$ Department of Chemistry, B. S. AbdurRahman University, Vandalaur, Chennai, \\ Tamilnadu-600048, India \\ alhajinmi@hotmail.com
}

Received 14 October 2012 / Accepted 12 November 2012

\begin{abstract}
Kinetics of oxidation of aniline by $N$-bromophthalimide (NBP) in acetonitrile-water solvent mixture at $303 \mathrm{~K}$ in the presence of perchloric acid has been followed iodometrically. The reaction is first order with respect to both aniline and NBP and is catalyzed by $\mathrm{H}^{+}$ion and the order of the reaction with respect to $\left[\mathrm{H}^{+}\right]$is also one. It has been found that the reaction rate is not affected by changes in ionic strength of the reaction medium or by the addition of acrylonitrile and potassium bromide. However, addition of phthalimide causes a decrease in the rate of reaction. An increase in the water content of the solvent mixture decreases the rate of reaction. Thermodynamic and activation parameters have also been evaluated.
\end{abstract}

Keywords: Oxidation, Kinetics, Mechanism, Aniline, $N$-Bromophthalimide

\section{Introduction}

Aromatic amines exhibit a complex behavior with different oxidants and yield a variety of complex and polymeric products. These products find application in drug and dye-stuff industries. Kinetics of oxidation of aromatic amines by a variety of oxidants have been studied extensively with $N$-halo compounds such as chloramine- $\mathrm{T}^{1,2}$, bromamine- $\mathrm{T}^{3}$,

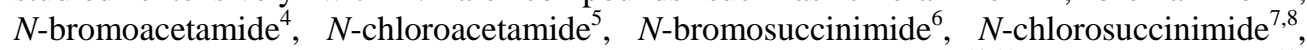
and $N$-iodosuccinimide ${ }^{9}$ and other oxidants such as peroxydisulfate ${ }^{10,11}$, hexacyanoferrate ${ }^{12}$, pyridinium chlorochromate ${ }^{13}$, sodium iodate ${ }^{14}$, isoquinolinium bromochromate ${ }^{15}$, imidazolium fluorochromate ${ }^{16}$, etc.

Among the various oxidants, the $N$-halo compounds get much attraction due to their ability to act as sources of halonium ions, hypohalite species and nitrogen anions, which act as both bases and nucleophiles. $N$-Bromphthlimide (NBP) is a potential oxidizing agent and 
has some definite advantages over other $N$-halo oxidants, which has been extensively used in the estimation of organic substrates ${ }^{17}$. A few reports of kinetics of oxidation of organic compounds such as amino acids ${ }^{18}$, sulfides ${ }^{19,20}$, hydroxy acids ${ }^{21}$, alcohols ${ }^{22}$ etc., using NBP are available in the literature. However, no detailed kinetic study of oxidation of aniline with $\mathrm{N}$-bromophthalimide has so far been attempted. We have investigated the kinetics of oxidation of aniline in acetonitrile-water mixture by NBP, to get a clear picture of mechanism of oxidation.

\section{Experimental}

All the chemicals used were of AR Grade. Anilines were distilled before use. NBP (E. Merck, GR) was used as such. All the solutions used in the study were made in distilled acetonitrile or doubly distilled water.

\section{Kinetic measurements}

The reactions were carried out under pseudo first order conditions by keeping an excess of substrate over NBP. The kinetic runs were carried out in 50\% acetonitrile-50\% water (v/v) solvent mixture in acid medium, the acid strength being maintained by the addition of $\mathrm{HClO}_{4}$ and the ionic strength by $\mathrm{NaClO}_{4}$. The progress of reaction was followed by monitoring the decrease in concentration of NBP iodometrically at suitable intervals of time. The rate constants were determined by least square method from the linear plots of log $[\mathrm{NBP}]$ versus time. Replicate runs showed that the rate constants were reproducible to within $\pm 5 \%$.

\section{Product analysis}

In a typical experiment, solutions of aniline and NBP in 1:2 molar ratio were mixed and kept aside for 2 to 3 days. The resulting precipitate was filtered off and washed with distilled water until free from NBP. TLC of the precipitate using benzene as an eluent revealed the presence of two compounds. The major product (85\%) was identified as azobenzene by mixed melting point determination with an authentic specimen.

\section{Stoichiometry}

In a typical experiment, a reaction mixture containing 10 times excess of NBP over aniline was prepared and allowed to react overnight. Then the unreacted NBP was estimated, which established a 1:1 stoichiometry between NBP and aniline, as represented in the following scheme.

$$
2 \mathrm{C}_{6} \mathrm{H}_{5} \mathrm{NH}_{2}+2 \mathrm{NBP} \rightarrow \mathrm{C}_{6} \mathrm{H}_{5}-\mathrm{N}=\mathrm{N}-\mathrm{C}_{6} \mathrm{H}_{5}+2 \mathrm{Phthalimide}+2 \mathrm{HBr}
$$

\section{Results and Discussion}

Oxidation of aniline by NBP has been conducted in $50 \%$ acetonitrile and $50 \%$ water medium at $303 \mathrm{~K}$, under pseudo first-order conditions and the result obtained were discussed in the following paragraphs. As it was found that ionic strength of the reaction medium had no significant effect on reaction rate, constancy of ionic strength was not maintained throughout the present study.

The values of $k_{\text {obs }}$ were calculated for different concentrations of NBP by maintaining other parameters at constant values. The data in Table 1 show that the rate constant remain almost constant on varying the concentration of NBP, revealing that the reaction follows a first-order kinetics with respect to [NBP]. The $k_{\text {obs }}$ values calculated for different concentrations of aniline by keeping the concentrations of NBP and $\mathrm{H}^{+}$at constant values are also included in Table 1 . The data establish that the rate increases with increase in [aniline] in a first-order fashion. Further, the plot of $k_{\text {obs }}$ versus [aniline] is excellently linear passing through origin (Figure $1 ; \mathrm{r}=0.999$ ). Also, the $k_{2}$ values remain constant when [aniline] 
is varied (Table 1); this result, coupled with the nearly unit slope value of the double logarithmic plot between $k_{\text {obs }}$ and [aniline] ( $\mathrm{r}=0.999$; slope $\left.=0.987 \pm 0.006\right)$ confirms the firstorder nature of the reaction with respect to [aniline].

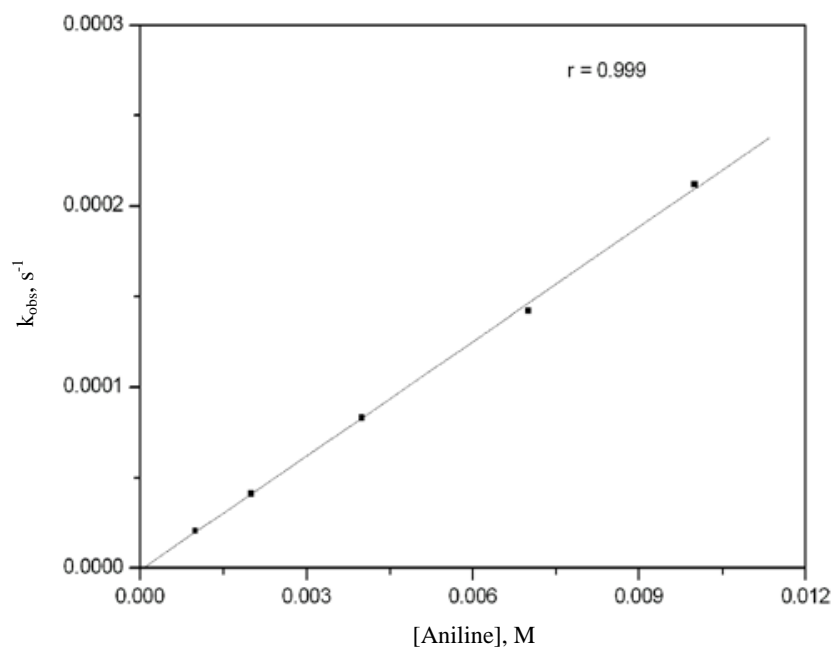

Figure 1. Direct plot of $k_{\text {obs }}$ versus [aniline] for the oxidation of aniline with NBP

The values of $k_{\mathrm{obs}}$ were calculated for different concentrations of $\mathrm{H}^{+}$by keeping the [NBP] and [aniline] at constant values. The $k_{\text {obs }}$ value increases linearly with increase in $\left[\mathrm{H}^{+}\right]$ (Table 1), establishing the catalytic behavior of perchloric acid. The double logarithmic plot of $k_{\text {obs }}$ versus $\left[\mathrm{H}^{+}\right]$is excellently linear $(\mathrm{r}=0.998$; slope $=1.03 \pm 0.03)$ with a slope value very equal to one, establishing that the reaction is first-order with respect to $\left[\mathrm{H}^{+}\right]$.

Table 1. Pseudo first-order and second-order rate constants for the oxidation of aniline by NBP in 50:50 acetonitrile-water (v/v) mixture at $303 \mathrm{~K}^{\mathrm{a}, \mathrm{b}}$

\begin{tabular}{ccccc}
\hline $10^{4}[\mathrm{NBP}], \mathrm{M}$ & $10^{3}$ [Aniline], $\mathrm{M}$ & $10^{3}\left[\mathrm{H}^{+}\right], \mathrm{M}$ & $10^{5} k_{\mathrm{obs}}, \mathrm{s}^{-1}$ & $10^{2} k_{2}^{\mathrm{c}}, \mathrm{M}^{-1} \mathrm{~s}^{-1}$ \\
\hline 1.0 & 1.0 & 1.0 & $2.06 \pm 0.15$ & $2.06 \pm 0.15$ \\
2.0 & 1.0 & 1.0 & $2.08 \pm 0.17$ & $2.08 \pm 0.17$ \\
3.0 & 1.0 & 1.0 & $2.02 \pm 0.16$ & $2.02 \pm 0.16$ \\
5.0 & 1.0 & 1.0 & $2.07 \pm 0.15$ & $2.07 \pm 0.15$ \\
7.0 & 1.0 & 1.0 & $2.10 \pm 0.18$ & $2.10 \pm 0.18$ \\
2.0 & 2.0 & 1.0 & $4.12 \pm 0.36$ & $2.06 \pm 0.18$ \\
2.0 & 4.0 & 1.0 & $8.32 \pm 0.71$ & $2.08 \pm 0.18$ \\
2.0 & 7.0 & 1.0 & $14.2 \pm 1.1$ & $2.03 \pm 0.16$ \\
2.0 & 10.0 & 1.0 & $21.2 \pm 1.9$ & $2.12 \pm 0.19$ \\
2.0 & 1.0 & 0.5 & $1.02 \pm 0.09$ & $1.02 \pm 0.09$ \\
2.0 & 1.0 & 2.0 & $4.28 \pm 0.38$ & $4.28 \pm 0.38$ \\
2.0 & 1.0 & 3.0 & $6.99 \pm 0.62$ & $6.99 \pm 0.62$ \\
2.0 & 1.0 & 5.0 & $10.4 \pm 0.97$ & $10.4 \pm 0.97$ \\
\hline
\end{tabular}

${ }^{a}$ As determined by iodometrically following the disappearance of NBP; the error quoted in $k$ values is the $95 \%$ confidence limit of 'Student $t$ test'. ${ }^{b}$ Estimated from pseudo first order plots over $80 \%$ reaction. 'Individual $k_{2}$ values estimated as $k_{\text {obs }}$ /[Aniline]。

The effect of ionic strength of the reaction on the reaction rate was studied by varying the ionic strength of the medium by adding different quantities of $\mathrm{NaClO}_{4}$, maintaining other 
parameters constant (Table 2). The data reveal that variation of ionic strength of the medium has no significant effect on reaction rate. This result shows the participation of a neutral species as a reactant in the rate-determining step. The kinetic runs carried out in the presence of acrylonitrile (Table 2) establish that the reaction fails to initiate the polymerisation of added acrylonitrile, which rules out the involvement of free radical intermediates. The kinetic runs carried out in the presence of $\mathrm{KBr}$ (Table 2) establish that the reaction rate does not change significantly with increasing concentration of added $\mathrm{KBr}$. This result rules out the participation of $\mathrm{Br}^{-}$or $\mathrm{Br}_{2}$ as reactive species in the present reaction.

Table 2. Influence of ionic strength, acrylonitrile (AN) and $\mathrm{KBr}$ on reaction rate for the oxidation of aniline by NBP in 1:1 acetonitrile-water (v/v) mixture at $303 \mathrm{~K}^{\mathrm{a}}$

\begin{tabular}{cccccc}
\hline$[\mathrm{I}], \mathrm{M}$ & $10^{4} k_{\mathrm{obs}}, \mathrm{s}^{-1}$ & {$[\mathrm{AN}], \mathrm{M}$} & $10^{4} k_{\mathrm{obs}}, \mathrm{s}^{-1}$ & {$[\mathrm{KBr}], \mathrm{M}$} & $10^{4} k_{\mathrm{obs}}, s^{-1}$ \\
\hline 0.30 & $2.08 \pm 0.17$ & 0 & $2.08 \pm 0.17$ & 0 & $2.08 \pm 0.17$ \\
0.50 & $2.03 \pm 0.18$ & 0.001 & $2.05 \pm 0.16$ & 0.001 & $2.04 \pm 0.17$ \\
0.75 & $2.12 \pm 0.19$ & 0.003 & $2.04 \pm 0.15$ & 0.003 & $2.05 \pm 0.18$ \\
1.00 & $2.15 \pm 0.20$ & 0.006 & $2.07 \pm 0.17$ & 0.006 & $2.01 \pm 0.16$ \\
\hline \multicolumn{5}{c}{${ }^{a}$ General conditions: $[\mathrm{NBP}]=0.0002 \mathrm{M}$; [aniline] $=0.001 \mathrm{M} ;\left[\mathrm{H}^{+}\right]=0.001 \mathrm{M}$}
\end{tabular}

The rate constant values measured for the oxidation of aniline with NBP in presence of differing amounts of phthalimide are listed in Table 3. The data show that the rate of reaction decreases significantly with increasing concentration of added phthalimide. The effect of the solvent composition on the reaction rate was studied by varying the acetonitrile content in the solvent mixture from $50 \%$ to $80 \%$ and the measured $k_{\text {obs }}$ values (Table 3 ) show that the rate of the reaction increases remarkably with increase in the percentage proportion of acetonitrile in the solvent medium, establishing that the reaction between aniline and NBP is favoured by a solvent of low dielectric constant. The plot of log $k_{\text {obs }}$ versus $1 / \mathrm{D}$ is linear, with positive slope, indicating that the reaction is of ion-dipole type.

Table 3. Influence of added phthalimide (NHP) and solvent polarity on reaction rate for the oxidation of aniline by NBP at $303 \mathrm{~K}^{\mathrm{a}}$

\begin{tabular}{cccc}
\hline$[\mathrm{NHP}], \mathrm{M}$ & $10^{4} k_{\mathrm{obs}}, \mathrm{s}^{-1}$ & $\% \mathrm{CH}_{3} \mathrm{CN}, \mathrm{v} / \mathrm{v}^{\mathrm{b}}$ & $10^{4} k_{\mathrm{obs}}, \mathrm{s}^{-1}$ \\
\hline 0 & $2.08 \pm 0.17$ & 0.50 & $2.08 \pm 0.17$ \\
0.001 & $2.01 \pm 0.17$ & 0.60 & $2.76 \pm 0.22$ \\
0.003 & $1.84 \pm 0.13$ & 0.70 & $3.93 \pm 0.37$ \\
0.006 & $1.62 \pm 0.11$ & 0.80 & $5.62 \pm 0.51$ \\
\hline
\end{tabular}

${ }^{a}$ General conditions: $[\mathrm{NBP}]=0.0002 \mathrm{M}$; $[$ Aniline $]=0.001 \mathrm{M} ;\left[\mathrm{H}^{+}\right]=0.001 \mathrm{M}$; Solvent $=1: 1$ acetonitrile-water $(\mathrm{v} / \mathrm{v})$ mixture unless otherwise stated. ${ }^{b}$ Rest was water

The reaction was carried out at three other temperatures (298, 313 and $323 \mathrm{~K})$ keeping other experimental conditions constant. From the Arrhenius and Eyring plots, the thermodynamic parameters for the oxidation of aniline were found to be $E_{a}=54.0 \pm 2.9 \mathrm{~kJ} / \mathrm{mol}, \Delta \mathrm{H}^{\ddagger}=51.4 \pm 2.1$ $\mathrm{kJ} / \mathrm{mol}, \Delta \mathrm{G}^{\ddagger}(303 \mathrm{~K})=84.0 \pm 4.1 \mathrm{~kJ} / \mathrm{mol}$ and $\Delta \mathrm{S}^{\ddagger}=-107.5 \pm 6.7 \mathrm{JK}^{-1} \mathrm{~mol}^{-1}$.

\section{Mechanism}

The reactive species that can be formed ${ }^{23}$ from $N$-halo compounds $(\mathrm{N}-\mathrm{X})$ in acidic solutions are $\mathrm{HOX}, \mathrm{H}_{2} \mathrm{O}^{+} \mathrm{X}$ and $>\mathrm{N}^{+} \mathrm{HX}$. The first-order dependence of present reaction on hydrogen ion concentration excludes the possibility of $>\mathrm{N}^{+} \mathrm{HBr}$ or $\mathrm{HOBr}$ being the reactive species and establishes the involvement of $\mathrm{H}_{2} \mathrm{O}^{+} \mathrm{Br}$, a stronger electrophile than $\mathrm{HOBr}$, as reactive species $^{24,25}$. The hypohalous acidium ion, $\mathrm{H}_{2} \mathrm{O}^{+} \mathrm{X}$ is the reactive species in the present study 
is further evidenced by the retarding effect shown by added phthalimide. The participation of $\mathrm{H}_{2} \mathrm{O}^{+} \mathrm{X}$ in many electrophilic and oxidation reactions has well been documented ${ }^{26,27}$. Based on the foregoing kinetic results, the following mechanism has been proposed.

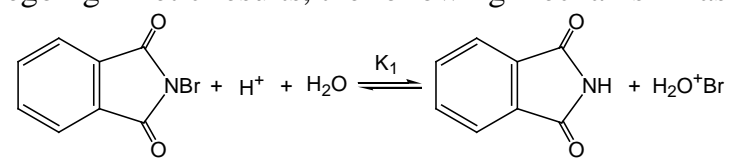

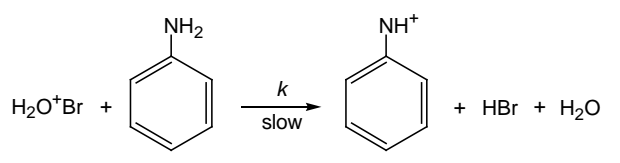<smiles></smiles>

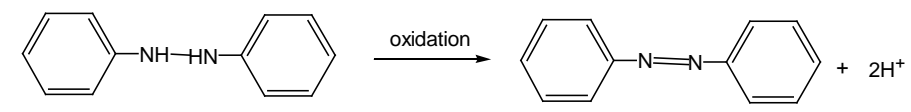

The rate law can be given as

$$
\text { Rate }=\frac{K_{1} k[\mathrm{NBP}][\text { Aniline }]\left[\mathrm{H}^{+}\right]}{[\mathrm{NHP}]}
$$

This rate law explains the first-order dependence of the reaction on [aniline], [NBP] and $\left[\mathrm{H}^{+}\right]$and the retarding effect of phthalimide.

$$
\begin{gathered}
k_{\mathrm{obs}}=\frac{K_{1} k[\text { Aniline }]\left[\mathrm{H}^{+}\right]}{[\mathrm{NHP}]} \\
k_{2}=\frac{K_{1} k\left[\mathrm{H}^{+}\right]}{[\mathrm{NHP}]}
\end{gathered}
$$

This equation explains the linear plot of $k_{2}$ versus $\left[\mathrm{H}^{+}\right]$which passes through origin (Figure 2).

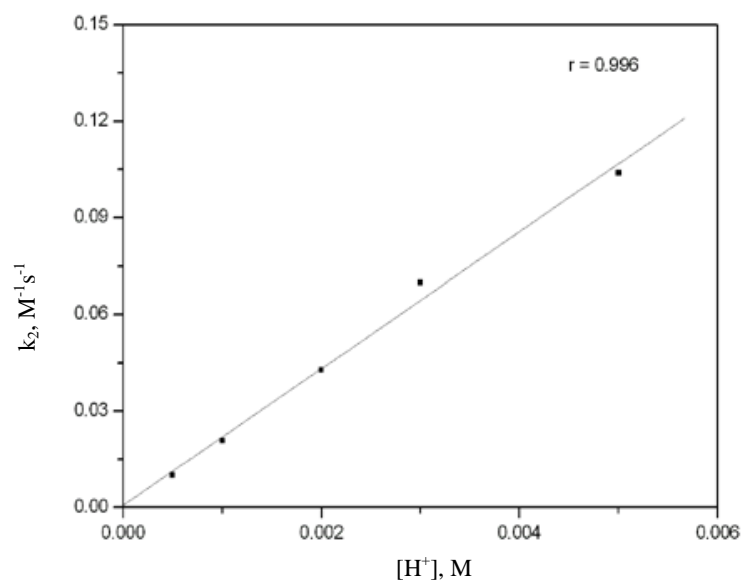

Figure 2. Plot of $k_{2}$ versus $\left[\mathrm{H}^{+}\right]$for the oxidation of aniline with NBP 


\section{Conclusion}

The NBP oxidation of aniline involves hypohalous acidium ion, $\mathrm{H}_{2} \mathrm{O}^{+} \mathrm{Br}$ as reactive species which attacks aniline electrophilically to give azobenzene as product. This mechanism is supported by the experimental data such as the reaction stoichiometry, the oxidation products and the activation parameters.

\section{Acknowledgement}

The authors thank the respective Managements for providing the necessary facilities.

\section{References}

1. Puttaswamy, Jagadeesh R V, Nirmala Vaz and Radhakrishna A, J Mol Catal:A Chem., 2005, 229, 211-220.

2. Radhakrishnamurty P S and Prasad Rao M D, Indian J Chem., 1976, 14B, 790.

3. $\quad$ Chanda B M, Vyas R and Landge S S, J Mol Catal A: Chem., 2004, 223, 57.

4. $\quad$ Sharadamani P R and Jagannadham V, Indian J Chem., 1990, 29A, 700.

5. Kumar A and Bhattacharjee G, Indian J Chem., 1991, 68, 523.

6. Radhakrishnamurty P S and Sahu S N, Indian J Chem., 1977, 15A, 788.

7. Radhakrishnamurty P S and Sahu S N, Indian J Chem., 1976, 14B, 898.

8. Nickson T E and Roche-Dolson C A, Synthesis, 1985, 669-670.

9. Das B and Kundu N G, Syn Commun., 1988, 18, 855-867.

10. Gupta R C and Srivastava S P, Indian J Chem., 1972, 10, 706.

11. Gric-Marjanavic G, Trchova M and Stejskal J, Int J Quantum Chem., 2008, 108, 318-333.

12. Radhakrishnamurty P S and Panda R K, Indian J Chem., 1971, 9, 1247.

13. Panigrahi G P and Mahapatro D D, Int J Chem Kinet., 1982, 14, 977-984.

14. Prasada Rao M D and Padmanabha J, Indian J Chem., 1980, 19A, 984.

15. Patwari S B, Khansole S V and Vibhute Y B, J Iran Chem Soc., 2009, 6, 399.

16. Bhuvaneshwari D S and Elango K P, Int J Chem Kinet., 2006, 38, 166-175.

17. Mohan Das C and Indrasenan P, J. Indian Chem Soc., 1987, 64, 382-384.

18. Singh A K, Jain B, Negi R, Katre Y and Sing S P, The Open Catal J., 2009, 2, 12

19. Alhaji N M I, Uduman Mohideen A M and Kalaimathi K, E-J Chem., 2011, 8(1), 1-8.

20. Alhaji N M I, Uduman Mohideen A M and Kalaimathi K, E-J Chem., 2011, 8(1), 159-166.

21. Thiagarajan V and Ramakrishnan S, Indian J Chem., 1998, 37B, 443.

22. Bharad J, Madje B and Unbale M, Int J Chem Tech Res., 2010, 2(4), 2150-2155.

23. Armesto X.L, Canle L M, Garcia M V and Santaballa J A, Chem Soc Rev., 1998, 27, 453-460.

24. Bishnoi M L and Banerji K K, Tetrahedron, 1985, 41, 6047-6050.

25. Thenraja D, Subramaniam P and Srinivasan C, J Chem Soc Perkin Trans 2, 2002, 2125-2156.

26. Waqar C, Singh B and Sharma J P, J Mol Catal., 1990, 60, 49.

27. Uma K V and Mayanna S M, Int J Chem Kinet., 1980, 12, 861-870. 